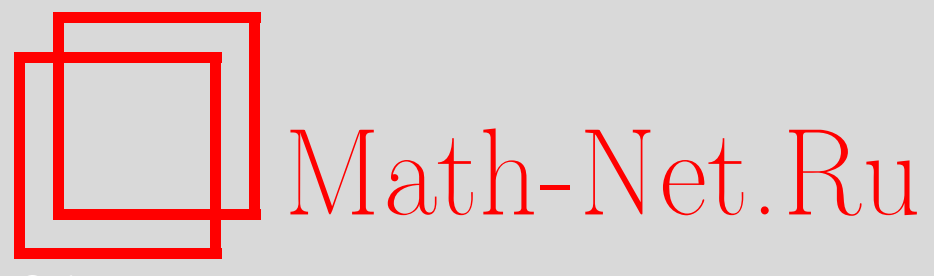

А. С. Братусь, М. В. Сафро, Асимптотика собственных значений матрицы Якоби систем полулинейных параболических уравнений, Матем. заметки, 2011, том 89, выпуск 2, 204-213

DOI: https://doi.org/10.4213/mzm8611

Использование Общероссийского математического портала Math-Net.Ru подразумевает, что вы прочитали и согласны с пользовательским соглашением http: //www . mathnet.ru/rus/agreement

Параметры загрузки:

IP: 54.147 .182 .235

26 апреля 2023 г., $17: 26: 50$

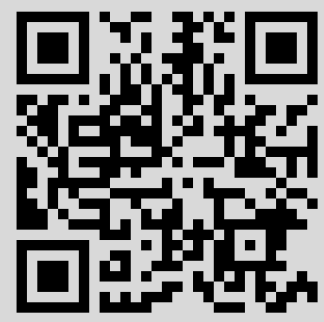


Том 89 выпуск 2 февраль 2011

\title{
Асимптотика собственных значений матрицы Якоби систем полулинейных параболических уравнений
}

\author{
А. С. Братусь, М. В. Сафро
}

Рассматриваются стационарные пространственно однородные решения системы полулинейных параболических уравнений в ограниченной области с краевыми условиями Неймана. Известно, что устойчивость таких решений связана со знаками вещественных частей собственных значений линеаризованного оператора, состоящего из матрицы Якоби динамической системы и дифференциального оператора, порожденного диффузионным процессом. Найдена асимптотика указанных собственных значений. Отдельно исследован случай, когда диффузионный оператор описывается матрицами, содержащими жордановы блоки, что соответствует случаю кросс-диффузии.

Библиография: 8 названий.

1. Постановка задачи. В ряде областей физики, химии, биологии [1]-[3] часто возникает задача об исследовании устойчивости стационарных решений краевых задач системы полулинейных параболических уравнений вида

$$
\frac{\partial u}{\partial t}=f(u)+D \Delta u
$$

Здесь $u=\left(u_{1}(x, t), \ldots, u_{n}(x, t)\right)$ - вектор-функция переменных $t \geqslant 0$ и $x_{1}, x_{2}, \ldots, x_{n}$, определенная при каждом $t$ в ограниченной области $\Omega \subset \mathbb{R}^{n}$ с гладкой границей $\partial \Omega$ при $t \geqslant 0, f(u)=\left(f_{1}(u), f_{2}(u), \ldots, f_{n}(u)\right)$ - гладкая дважды дифференцируемая вектор-функция, $D$ - матрица с неотрицательными элементами $d_{i j} \geqslant 0, i, j=1, \ldots, n$ (матрица диффузии); $\Delta-n$-мерный оператор Лапласа $\Delta u=\left(\Delta u_{1}, \Delta u_{2}, \ldots, \Delta u_{n}\right)$.

Заданы начальные условия

$$
u(x, 0)=\phi(x), \quad x \in \Omega .
$$

На границе $\partial \Omega$ области $\bar{\Omega}$ задано условие Неймана

$$
\left.\frac{\partial u}{\partial n}\right|_{\partial \Omega}=0
$$

Задача (1.1)-(1.3) изучается в полном метрическом пространстве вектор функций

$$
U=\left\{u_{1}(x, t), \ldots, u_{n}(x, t): u_{i}(x, t) \in H^{1}(\Omega), i=1, \ldots, n, x \in \Omega\right\},
$$


где $H^{1}(\Omega)$ - пространство функций Соболева с нормой

$$
\|u\|_{H^{1}}=\left(\sum_{i=1}^{n} \int_{\Omega}\left|\frac{\partial \nu}{\partial x_{i}}\right|^{2} d x\right)^{1 / 2}
$$

Наряду с системой (1.1) рассмотрим динамическую систему в $\mathbb{R}^{n}$

$$
\frac{d u}{d t}=f(u)
$$

Пусть $u^{0}=u_{0}^{1}, \ldots, u_{0}^{n}-$ стационарная точка системы (1.4), т.е. $f\left(u^{0}\right)=0$. Через $A$ обозначим матрицу Якоби вектор функции $f(u)$ в точке $u^{0}$

$$
A=\left(\frac{\partial f_{i}}{\partial u_{j}}\left(u^{0}\right)\right), \quad i, j=1, \ldots, n .
$$

Неподвижная точка $u^{0}$ системы (1.4) является также пространственно-однородным решением системы (1.1), поскольку выполняется равенство

$$
f\left(u^{0}\right)+D \Delta u^{0}=0 .
$$

Рассмотрим линейное приближение системы (1.1) в точке $u^{0}$ :

$$
\frac{d u}{d t}=A u+D \Delta u
$$

Согласно теореме об устойчивости по линейному приближению [1] система полулинейных параболических уравнений (1.1) будет устойчива в окрестности неподвижной точки $u^{0}$, если все собственные значения краевой задачи

$$
\begin{gathered}
A u+D \Delta u=\lambda u, \quad x \in \Omega, \\
\left.\frac{\partial u}{\partial n}\right|_{\partial \Omega}=0
\end{gathered}
$$

отрицательны. Если хотя бы одно собственное значение задачи (1.5), (1.6) положительно, (1.1) будет неустойчивой.

Известно, что даже если система (1.4) устойчива в окрестности решения $u=u^{0}$, то соответствующая система с диффузией может быть неустойчивой в окрестности этого пространственно-однородного решения [2] и наоборот. Поэтому (особенно в случае систем большой размерности) большую роль играет асимптотика собственных значений системы (1.5), (1.6). Заметим, что хотя знаки вещественных частей первого и последующих приближения собственных значений системы (1.1) не дают ни достаточного, ни необходимого условия устойчивости системы (1.1) в стационарной точке $u=u^{0}$, они являются важными признаками возможного поведения системы в окрестности этого решения.

2. Случай матрицы диффузии простой структуры. Пусть матрица $D$ имеет простую структуру, т.е. кратность любого собственного значения матрицы $D$ равна числу линейно независимых собственных векторов, отвечающих этому собственному значению. Предполагается, что все собственные значения матрицы $D$ 
неотрицательны. В этом случае существует невырожденная матрица $T$ такая, что $\Lambda=T^{-1} D T$, где $\Lambda$ - диагональная матрица, элементы которой - собственные числа матрицы $D$.

Положим в (1.5), (1.6)

$$
u(x)=T v(x), \quad v(x)=\left(v_{1}(x), \ldots, v_{n}(x)\right),
$$

тогда имеем

$$
\begin{gathered}
B v+\Lambda \Delta v=\lambda v \\
(v)_{\partial \Omega}=0,
\end{gathered}
$$

где $B=T^{-1} A T, x \in \Omega$. Рассмотрим решение краевой задачи на собственные значения для функции $Z(x), x \in \Omega$,

$$
\Delta Z=-\mu Z, \quad x \in \Omega, \quad \mu>0,\left.\quad \frac{\partial Z}{\partial n}\right|_{\partial \Omega}=0 .
$$

Известно, что эта задача имеет счетное число решений - собственных значений

$$
0=\mu_{0}<\mu_{1} \leqslant \cdots \leqslant \mu_{k} \leqslant \cdots, \quad \mu_{k} \rightarrow+\infty, \quad k \rightarrow+\infty,
$$

и соответствующее число собственных функций $\left\{Z_{k}(x)\right\}_{k=1}^{\infty}$, которые образуют полную ортогональную систему в $H^{1}(\Omega)[4]$.

Положим

$$
v(x)=y Z_{k}(x), \quad k=1,2, \ldots,
$$

где $y=\left(y_{1}, y_{2}, \ldots, y_{n}\right)$ - вектор в $\mathbb{C}^{n}$. Подставляя это равенство в $(2.1)$, придем к матричной задаче на собственные значения

$$
\left(B-\mu_{k} \Lambda\right) y=\lambda y, \quad k=1,2, \ldots .
$$

Положим $\varepsilon_{k}=\left(\mu_{k}\right)^{-1}, \varepsilon_{k} \rightarrow 0, k \rightarrow \infty$, тогда последнее равенство можно записать в виде

$$
\left(-\Lambda+\varepsilon_{k} B\right) y=\lambda\left(\varepsilon_{k}\right) y
$$

Здесь $\lambda\left(\varepsilon_{k}\right)=\lambda \varepsilon_{k}$.

Задача (2.4) может быть интерпретирована как задача об отыскании $n$ собственных значений $\lambda^{i}\left(\varepsilon_{k}\right)$ возмущенной посредством добавки матрицы $\varepsilon_{k} B$ к диагональной матрице $-\Lambda$.

Пусть $d_{1}, \ldots, d_{n}$ - элементы диагональной матрицы $\Lambda$, т.е. собственные числа исходной матрицы $D$ и $d_{i} \neq d_{j}$, а $b_{i j}$ - элементы матрицы $B$. Согласно результатам [5] собственные значения $\lambda^{i}\left(\varepsilon_{k}\right)$ и собственные векторы $y^{i}\left(\varepsilon_{k}\right), k=1,2, \ldots, n$, могут быть представлены в виде регулярного аналитического разложения по степеням $\varepsilon_{k}$ с некоторыми коэффициентами:

$$
\begin{array}{cl}
\lambda\left(\varepsilon_{k}\right)=-d_{i}+\varepsilon_{k} \eta_{1}^{i}+\varepsilon_{k}^{2} \eta_{2}^{i}+o\left(\varepsilon_{k}^{2}\right), & y^{i}\left(\varepsilon_{k}\right)=y^{i, 0}+\varepsilon_{k} y^{i, 1}+\varepsilon_{k}^{2} y^{i, 2}+o\left(\varepsilon_{k}^{2}\right), \\
i=1, \ldots, n, & k=1,2, \ldots .
\end{array}
$$

Легко заметить, что $y^{i, 0}$ является вектором, у которого все компоненты нули, кроме $i$-й, равной единице. 
Подставляя разложения (2.5) в (2.4) и собирая члены одного и того же порядка по $\varepsilon_{k}$, имеем

$$
\begin{aligned}
-\Lambda y^{i, 0} & =-d_{i} y^{i, 0}, \\
-\Lambda y^{i, 1}+B y^{i, 0} & =\eta_{1}^{i} y^{i, 0}-d_{i} y^{i, 1}, \\
-\Lambda y^{i, 2}+B y^{i, 1} & =\eta_{2}^{i} y^{i, 0}+\eta_{1}^{i} y^{i, 1}-d_{i} y^{i, 2} .
\end{aligned}
$$

Умножим скалярно обе части второго равенства из $(2.6)$ на $y^{i, 0}$, получим

$$
\eta_{1}^{i}=\left(B y^{1,0}, y^{i, 0}\right)=b_{i i}, \quad i=1, \ldots, n .
$$

Для отыскания следующего члена разложения $\eta_{2}^{i}$ представим вектор $y^{i, 1}$ в виде линейной комбинации базисных векторов $y^{i, 0}$ :

$$
y^{i, 1}=\sum_{j=1}^{n} y^{j, 1} c_{j}^{i}, \quad c_{j}^{i}=\text { const. }
$$

Подставляя это разложение во второе из равенств (2.6) и умножая последовательно обе части на векторы $y^{1,0}, y^{2,0}, \ldots, y^{n, 0}$, получим

$$
c_{j}^{i}=\frac{\left(B y^{i, 0}, y^{i, 0}\right)}{d_{i}-d_{j}}=-\frac{b_{j i}}{d_{i}-d_{j}}, \quad i \neq j .
$$

Коэффициенты $c_{i}^{i}, i=1, \ldots, n$, остаются неизвестными, однако непосредственно проверяется, что на дальнейшие выкладки это обстоятельство никак не влияет. С учетом последнего равенства имеем

$$
y^{i, 1}=-\sum_{j \neq i} \frac{b_{j i}}{d_{i}-d_{j}} y^{i, 0}+\sum_{i=1}^{n} c_{i}^{i} y^{1,0} .
$$

Подставляя теперь это равенство в третье уравнение (2.6) и умножая обе части скалярно на $y^{i, 0}$, получим

$$
\eta_{2}^{i}=-\sum_{i \neq j} \frac{b_{j i}}{d_{i}-d_{j}}\left(B y^{j, 0}, y^{i, 0}\right)=-\sum_{j \neq i} \frac{b_{j i} b_{i j}}{d_{i}-d_{j}} .
$$

В итоге доказано следующее

УТВЕРЖДЕНИЕ 1. Пусть матрица диффузии $D$ в (1.1) имеет простую струкmуру, и пусть $d_{i}, i=1, \ldots, n,-$ собственные значения матрицы $D$, причем $d_{i} \neq d_{j}$, $i \neq j$. Пусть $\mu_{k}, k=1,2, \ldots,-$ собственные значения краевой задачи $(2.3), \mu_{k} \rightarrow \infty$, $k \rightarrow \infty, a b_{i j}, i, j=1, \ldots, n,-$ элементы матрищы $B$. Тогда собственные значения задачи (1.5), (1.6) могут быть представлены в виде асимптотического разложения

$$
\begin{gathered}
\lambda_{k}^{i}=-d_{i} \mu_{k}+b_{i i}-\mu_{k}^{-1} \sum_{i \neq j} \frac{b_{i j} b_{j i}}{d_{i}-d_{j}}+O\left(\mu_{k}^{-2}\right), \quad i=1, \ldots, n, \quad k=1,2, \ldots, \\
\mu_{k} \rightarrow \infty, \quad k \rightarrow \infty .
\end{gathered}
$$


ЗАмечаниЕ 1. Формула (2.7) показывает, что первые члены разложения (2.7) будут положительны, если $d_{i}<b_{i i} / \mu_{k}, b_{i i}>0$, т.е. если коэффициенты диффузии достаточно малы. Факт потери устойчивости пространственно-однородных решений ${ }^{1}$ и возникновение пространственно-неоднородных состояний диссипативных структур равновесия при малых коэффициентах диффузии описан в [7]. Причем при последовательном уменьшении коэффициентов диффузии возникают бифуркации (перестройки) структуры диссипативных членов.

В связи с этим возникает гипотеза, что достаточным условием возникновения диссипативных структур является условие $d_{i}<c / \mu_{1}, i=1, \ldots, n$, здесь $\mu_{1}$ - первое ненулевое значение, $c$ - const $>0$, а появление бифуркационных решений определяется неравенством $d_{i}<c_{i} / \mu_{k}, k=2,3, \ldots, c_{i}$ - const $>0$. Отметим, что в частных случаях это условие является верным [8].

\section{3. Случай, когда матрица диффузии имеет кратные собственные зна-} чения. Пусть среди собственных значений матрицы $D$ имеются кратные. Например, $d_{1}$ - значение кратности $s \geqslant 2$. Поскольку $D$ - матрица простой структуры, то этому кратному собственному значению отвечают $s$ линейно-независимых собственных векторов. Не умаляя общности, можно сказать, что матрица $\Lambda$ имеет по диагонали $s$ раз повторяющееся собственное значение $d_{1}$ в левом верхнем углу. Остальные собственные значения $d_{2}, \ldots, d_{r}(r=n-s)$ будем считать простыми и не равными $d_{1}$. Собственные векторы, отвечающие кратному значению $d_{1}$, имеют вид

$$
\omega^{1}=(1,0, \ldots,), \quad \omega^{2}=(0,1,0, \ldots, 0), \quad \ldots, \quad \omega^{s}=(0, \ldots, 0,1,0, \ldots, 0)
$$

(в $\omega^{s}$ единица стоит на $s$-м месте). Собственные векторы, отвечающие остальным собственным значениям, обозначим, как и ранее, через $y^{0, i}, i=s+1, \ldots, n-s$ (единица - на $s$-м месте). Возмущенное собственное значение $\eta^{1}\left(\xi_{k}\right)$ задачи $(2.4)$ можно [5] искать в виде первого равенства (2.5) с $i=1$. Представим собственный вектор $y^{1}\left(\xi_{k}\right)$ в виде

$$
y^{1}\left(\xi_{k}\right)=\sum_{j=1}^{s} k_{j} \omega^{j}+\varepsilon_{k} y^{1,1}+\varepsilon_{k}^{2} y^{1,2}+o\left(\varepsilon_{k}^{2}\right) .
$$

Здесь $k_{j}$ - некоторые постоянные, вообще говоря, комплексные, а $\omega^{j}$ определены в (3.1). Подставляя (3.2) в (2.4) и собирая члены с одинаковыми степенями $\varepsilon_{k}$, получим

$$
\begin{aligned}
\left(-\Lambda+d_{1}\right) y^{1,1}+\sum_{j=1}^{s} k_{j} B \omega^{j} & =\eta_{1}^{1} \sum_{j=1}^{s} k_{j} \omega^{j}+\eta_{1}^{1} y^{1,1}, \\
s\left(-\Lambda+d_{1}\right) y^{1,2}+B y^{1,1} & =\eta_{2}^{1} \sum_{j=1}^{s} k_{j} \omega^{j}+\eta_{1}^{1} y^{1,1} .
\end{aligned}
$$

Умножая последовательно обе части первого равенства (3.3) скалярно на векторы $\omega^{r}, r=1, \ldots, n$, получим систему линейных однородных уравнений относительно постоянных $k_{j}$ :

$$
\sum_{j=1}^{s} b_{r j} k_{j}=k_{r} \eta_{1}^{1}, \quad r=1, \ldots, s .
$$

\footnotetext{
${ }^{1}$ Пространственно-однородным решением системы называется решение $\bar{u}(x)$ уравнения $\Delta \bar{u}+$ $f(\bar{u})=0, \bar{u}(x) \neq$ const.
} 
Эта система имеет решение тогда и только тогда, когда

$$
\left|B_{s}-\eta_{1}^{1} E\right|=0 .
$$

Здесь $B_{s}-(s \times s)$-матрица с элементами $b_{i j}, i, j=1, \ldots, s$. Следовательно, величины $\eta_{1}^{1}-$ собственные значения матрицы $B_{s}$. Обозначим эти значения через $\left(\eta_{1}^{1}\right)_{p}$, $p=1, \ldots, s$. Вектор $y^{1,1}$ будем искать в виде

$$
y^{1,1}=\sum_{j=1}^{s} c_{j} \omega^{j}+\sum_{j=s+1}^{n} c_{j} y^{0, j}, \quad c_{j}=\text { const. }
$$

Если это равенство подставить в первое уравнение (3.3), а затем умножить обе его части скалярно на векторы $y^{0, j}, j=s+1, \ldots, n$, то получим

$$
c_{j}=-\frac{\sum_{j=1}^{s} k_{j} b_{i j}}{d_{1}-d_{i}}, \quad i=s+1, \ldots, n .
$$

Постоянные $c_{j}, j=1, \ldots, s$, при этом не определяются. В итоге имеет место разложение

$$
y^{1,1}=\sum_{j=1}^{s} c_{j} \omega^{j}-\sum_{j=s+1}^{n} \frac{\sum_{j=1}^{s} k_{j} b_{i j}}{d_{1}-d_{i}} y^{0, j} .
$$

Подставим это равенство во второе уравнение (3.3) и умножим его скалярно на вектор

$$
\bar{y}^{1,0}=\sum_{j=1}^{s} \bar{k}_{j} \omega^{j}
$$

Окончательно получим

$$
\sum_{j=1}^{s} \sum_{i=s+1}^{n} \frac{\left(\sum_{r=1}^{s} k_{r} b_{r i}\right) \bar{k}_{j} b_{j i}}{d_{1}-d_{i}}+\sum_{i, r=1}^{s} b_{j r} c_{r} \bar{k}_{j}=\eta_{1}^{1} \sum_{j=1}^{s} c_{j} \bar{k}_{j}+\eta_{1}^{2} \sum_{r=1}^{s}\left|k_{r}^{p}\right|^{2} .
$$

Следовательно, для величин $\left(\eta_{2}^{1}\right)_{p}$ имеет место разложение

$$
\left(\eta_{2}^{1}\right)_{p}=-\sum_{j=1}^{s} \sum_{i=s+1}^{n} \frac{\left(\sum_{r=1}^{s} k_{r}^{p} b_{r i}\right) \bar{k}_{j}^{p} b_{j i}}{\left(d_{1}-d_{i}\right) \sum_{r=1}^{s}\left|k_{r}^{p}\right|^{2}}, \quad p=1, \ldots, s .
$$

Здесь $k_{j}^{p}$ - координаты $p$-го собственного вектора задачи $B_{s} k=\eta_{1}^{1} k$, отвечающего собственному значению $\left(\eta_{1}^{1}\right)_{p}, p=1, \ldots, s$.

Полученный результат можно сформулировать в следующем виде.

УтВеРЖДЕНИЕ 2. Пусть матрица $D$ в (1.1) имеет простую структуру $u d_{1}-$ собственное значение кратности s. Тогда отвечающие этому собственному значению s собственных значений задачи (1.5), (1.6) представляются в виде асимптотического разложения

$$
\lambda_{k}^{p}=-d_{1} \mu_{k}+\left(\eta_{1}^{1}\right)_{p}-\mu_{k}^{-1} \sum_{j=1}^{s} \sum_{i=s+1}^{n} \frac{\left(\sum_{r=1}^{s} k_{r}^{p} b_{r i}\right) \bar{k}_{j}^{p} b_{j i}}{\left(d_{1}-d_{i}\right) \sum_{r=1}^{s}\left|k_{r}^{p}\right|^{2}}+O\left(\mu_{k}^{-2}\right) .
$$

Здесъ $\left(\eta_{1}^{1}\right)_{p}$ - p-е собственное значение задачи $(3.3), k_{j}^{p}$ - координаты собственного вектора, отвечающего собственному значению $\left(\eta_{1}^{1}\right)_{p}, p=1, \ldots, s$. Остальные собственные значения выражаются формулой (2.7) при $s=1, \ldots, n$, где $d_{j}=d_{1}$ для $j=1, \ldots, s$. 
ЗАмЕчаниЕ 2 . Если $d_{1}$ - собственное число кратности $n$, то формула (3.3) приобретает вид точного равенства:

$$
\lambda_{k}^{p}=-d_{1} \mu_{k}+\left(\eta_{1}^{1}\right)_{p}, \quad p=1, \ldots, n,
$$

где $\left(\eta_{1}^{1}\right)_{p}$ - собственное значение матрицы $B$.

4. Случай, когда матрица диффузии имеет жордановы блоки. Рассмотрим случай, когда матрица $\Lambda$ содержит жорданов блок, что соответствует наличию кросс-диффузионных членов в системе

$$
J_{m}=\left(\begin{array}{cccccc}
d_{1} & 1 & 0 & \ldots & 0 & 0 \\
0 & d_{1} & 1 & \ldots & 0 & 0 \\
\ldots & \ldots & \ldots & \ldots & \ldots & \ldots \\
0 & 0 & 0 & \ldots & d_{1} & 1 \\
0 & 0 & 0 & \ldots & 0 & d_{1}
\end{array}\right)
$$

Пусть $d_{m+1}, d_{m+2}, \ldots, d_{n}$ - остальные собственные значения матрицы $D ; m$-кратному собственному значению $d_{1}$ отвечает единственный собственный вектор $y^{1,0}$, а остальным собственным значениям - собственные векторы $y^{i, 0}, i=m+1, \ldots, n$. Известно, что в этом случае собственное значение $\lambda_{k}^{1}\left(\varepsilon_{k}\right)$ и собственные векторы $y^{1}\left(\varepsilon_{k}\right)$ возмущенной задачи представляются по степеням $\varepsilon_{k}^{1 / m}[5],[6]$ :

$$
\begin{aligned}
& \lambda^{1}\left(\varepsilon_{k}\right)=-d_{1}+\varepsilon_{k}^{1 / m} \eta_{1}^{1}+\varepsilon_{k}^{2 / m}+\varepsilon_{k}^{2 / m} \varepsilon_{k}^{3 / m} \eta_{3}^{1}+o\left(\varepsilon_{k}^{3 / m}\right), \\
& y^{1}\left(\varepsilon_{k}\right)=-y^{1,0}+\varepsilon_{k}^{1 / m} y^{1,1}+\varepsilon_{k}^{2 / m} y^{1,2}+\varepsilon_{k}^{3 / m} y^{1,3}+o\left(\varepsilon_{k}^{3 / m}\right) .
\end{aligned}
$$

Остальные собственные векторы и собственные значения представляются в виде разложения (2.5) по целым степеням $\varepsilon_{k}$.

Рассмотрим случай жорданова блока

$$
J_{2}=\left(\begin{array}{cc}
d_{1} & 1 \\
0 & d_{1}
\end{array}\right)
$$

В этом случае разложение (4.1) реализуется по степеням $\varepsilon_{k}^{1 / 2}$.

Если подставить (4.1) в (2.4) и собрать члены одного порядка по $\varepsilon_{k}$, то получим цепочку равенств

$$
\begin{aligned}
\left(-\Lambda+d_{1}\right) y^{1,0} & =0 \\
\left(-\Lambda+d_{1}\right) y^{1,1} & =\eta_{1}^{1} y^{1,0} \\
\left(-\Lambda+d_{1}\right) y^{1,2}+B y^{1,0} & =\eta_{1}^{1} y^{1,1}+\eta_{2}^{1} y^{1,0} \\
\left(-\Lambda+d_{1}\right) y^{1,2}+B y^{1,1} & =\eta_{1}^{1} y^{1,2}+\eta_{2}^{1} y^{1,1}+\eta_{3}^{1} y^{1,0} \\
\left(-\Lambda+d_{1}\right) y^{1,3}+B y^{1,1} & =\eta_{1}^{1} y^{1,3}+\eta_{2}^{1} y^{1,2}+\eta_{3}^{1} y^{1,1}+\eta_{4}^{1} y^{1,0}
\end{aligned}
$$

где $y^{1,0}=(1,0, \ldots, 0)$ и $y^{i, 0}=(0, \ldots, 1, \ldots, 0)$ ( 1 стоит на $i$-м месте), $i=3, \ldots, n$. Второе уравнение системы (4.2) разрешимо, если и только если его правая часть ортогональна к решениям сопряженного уравнения

$$
\left(-\Lambda^{*}+d_{1}\right) z^{0}=0
$$


Это условие выполняется, когда $z^{0}=(0,1,0, \ldots, 0)$. Решением второго уравнения системы (4.2) является $y^{1,1}=\left(\alpha_{1}, \eta_{1}^{1}, 0, \ldots, 0\right)$, где $\alpha_{1}$ - произвольная константа. Умножая третье уравнение системы (4.2) на $z^{0}$ и используя уравнение (4.3), получаем

$$
\begin{aligned}
\left((-\Lambda+d) y^{1,2}, z^{0}\right) & =\left(y^{1,2},\left(-\Lambda^{*}+\bar{d}\right) z^{0}\right)=0 \\
-b_{21}-\left(\eta_{1}^{1}\right)^{2} & =0, \quad \eta_{1}^{1}= \pm \sqrt{-b_{21}} .
\end{aligned}
$$

Положим $y^{1,2}=\left(\alpha_{1}, \beta_{2}, \gamma_{3}, \ldots, \gamma_{n}\right)$. Первая компонента третьего уравнения системы (4.2) дает $-\beta_{2}+b_{11}=\eta_{2}^{1}+\alpha_{1} \eta_{1}^{1}$, и $i$-я компонента дает $\gamma_{i}=-b_{i 1} /\left(d_{i}-d_{1}\right)$, $i=3, \ldots, n$, где $d_{i} \neq d_{1}$. Умножая четвертое уравнение системы (4.2) на $z^{0}$ получаем соотношения к уравнению (4.4) для вектора $y^{1,3}$ и формулу $\left(y^{1,0}, z^{0}\right)=0$, $-b_{21} \alpha_{1}+\eta_{1}^{1} b_{22}-2 \eta_{2}^{1} \eta_{1}^{1}+\eta_{1}^{1} b_{11}-\alpha_{1} \eta_{1}^{2}=0$.

Подставив $\left(\eta_{1}^{1}\right)^{2}=-b_{21}$, получим

$$
\eta_{2}^{1}=\frac{b_{11}+b_{22}}{2} .
$$

Вернемся к четвертому уравнению системы (4.2) и положим $y^{1,3}=\left(\alpha_{3}, \beta_{3}, \gamma_{3}^{\prime}, \ldots, \gamma_{n}^{\prime}\right)$. Тогда первая компонента этого уравнения дает

$$
\beta_{3}=b_{11} \alpha_{1}+b_{12} \eta_{1}^{1}-\eta_{3}^{1}-\alpha_{1} \eta_{2}^{1}-\alpha_{2} \eta_{1}^{1} .
$$

Умножая пятое уравнение системы (4.2) на $z^{0}$ и используя формулу (4.4) для вектора $y^{1,4}$, получим

$$
\begin{aligned}
& -b_{21} \alpha_{2}-b_{22}\left(b_{11}-\eta_{2}^{1}-\alpha_{1} \eta_{1}^{1}\right)-\eta_{1}^{1} \eta_{3}^{1}+b_{11} \eta_{2}^{1}-\left(\eta_{1}^{1}\right)^{2} \\
& \quad-2 \alpha_{1} \eta_{1}^{1} \eta_{2}^{1}+\eta_{1}^{1} b_{11} \alpha_{1}+b_{12}\left(\eta_{1}^{1}\right)^{2}-\eta_{1}^{1} \eta_{3}^{1}-\left(\eta_{1}^{1}\right)^{2} \alpha_{2}=0 .
\end{aligned}
$$

Из (4.5), (4.6) следует

$$
\alpha_{1}\left(b_{22}+b_{11}-2 \eta_{2}^{1}\right) \eta_{1}^{1}=0, \quad \alpha_{2}\left(-b_{21}-\left(\eta_{1}^{1}\right)^{2}\right)=0 .
$$

Поэтому

$$
2 \eta_{1}^{1} \eta_{3}^{1}=\frac{\left(b_{11}+b_{22}\right)^{2}}{4}-b_{22} b_{11}+b_{12} b_{21}=\frac{\left(b_{11}-b_{22}\right)^{2}+4 b_{12} b_{21}}{4} .
$$

Так же, как в предыдущем случае, можно единственным образом определить $\gamma_{i}^{\prime}$, $3, \ldots, n$, рассматривая $i$-ю компоненту пятого уравнения системы (4.2).

УтвеРжДЕНИЕ 3. Предположим, что у канонической формы матрицы D имеется простой жордановый блок

$$
\left(\begin{array}{cc}
d_{1} & 1 \\
0 & d_{1}
\end{array}\right)
$$

Если $b_{21} \neq 0$, то соответствующие собственные значения задач (1.5), (1.6) имеют борму

$$
\begin{aligned}
\lambda_{k}^{1,2}=- & d_{1} \mu_{k} \pm\left(\mu_{k}\right)^{1 / 2} \sqrt{-b_{21}}+\frac{b_{11}+b_{22}}{2} \\
& \pm \frac{\left(\mu_{k}\right)^{-1 / 2}}{8\left(-b_{21}\right)^{1 / 2}}\left[\left(b_{11}-b_{22}\right)^{2}+4 b_{12} b_{21}\right]+o\left(\mu_{k}^{-1 / 2}\right),
\end{aligned}
$$

где знаки "+" и“-" определяются для значений $\lambda_{k}^{1} u \lambda_{k}^{2}$ соответственно. 
Случай $b_{21}=0$ является вырожденным. Нетрудно показать, что в этом случае $\lambda_{k}^{i}=-d_{1} \mu_{k}+b_{i i}, k=1,2, \ldots, i=1,2$. Аналогично доказываются формулы для жордановых матриц третьего и четвертого порядков.

УтвеРжДЕНИЕ 4. Если матрица $\Lambda$ содержит жорданов блок третъего или четвертого порядка, то имеют место соответственно следующие представления:

$$
\begin{gathered}
\left(\lambda_{k}^{1}\right)_{i}=-d_{1} \mu_{k}+\left(\mu_{k}\right)^{2 / 3}\left(b_{31}\right)_{i}^{1 / 3}-\left(\mu_{k}\right)^{1 / 3} \frac{b_{21}+b_{32}}{3\left(b_{31}\right)_{i}^{1 / 3}}+\left(\frac{b_{11}+b_{22}+b_{33}}{3}\right)+o\left(\left(\mu_{k}\right)^{-1 / 3}\right), \\
k=1,2, \ldots, \quad i=1,2,3,
\end{gathered}
$$

где $\left(b_{31}\right)_{i}^{1 / 3}$ - кубический корень элемента $b_{31} \neq 0$ матрицы $B$,

$$
\begin{gathered}
\left(\lambda_{k}^{1}\right)_{i}=-d_{1} \mu_{k}+\left(\mu_{k}\right)^{3 / 4}\left(-b_{41}\right)_{i}^{1 / 4}-\left(\mu_{k}\right)^{1 / 2} \frac{b_{42}+b_{31}}{\left(-b_{41}\right)_{i}^{1 / 4}}+o\left(\left(\mu_{k}\right)^{-1 / 4}\right), \\
k=1,2, \ldots, \quad i=1,2,3,
\end{gathered}
$$

где $\left(-b_{41}\right)_{i}^{1 / 4}$ - корни четвертой степени элемента $b_{41} \neq 0$ матрицы $B$.

ЗАмечАнИЕ 3. Случаи $b_{31}=0$ и $b_{41}=0$ являются вырожденными. В этих случаях разложение (4.1) содержит целое число $\varepsilon_{k}$, причем

$$
\begin{array}{ll}
\lambda_{k}^{1}=-\mu_{k} d_{1}+\frac{b_{11} b_{32}+b_{21} b_{33}}{b_{21}}+O\left(\mu_{k}^{-1}\right), & n=3, \\
\lambda_{k}^{1}=-\mu_{k} d_{1}+\frac{b_{42}+b_{43} b_{21}+b_{23} b_{44}}{b_{23}}+O\left(\mu_{k}^{-1}\right), & n=4 .
\end{array}
$$

ЗАмечАние 4. Можно показать, что для случаев $n=5,6, \ldots$ главная часть асимптотического разложения имеет вид $\left(-b_{n 1}\right)^{1 / n}$, если $n=2 k$ или $\left(b_{n 1}\right)^{1 / n}$, если $n=2 k-1, k=1,2, \ldots$. Поэтому имеет место следующее представление:

$$
\lambda_{k}^{i}=-d_{1} \mu_{k}+\left( \pm b_{n 1}\right)^{1 / n} \mu_{k}^{1-1 / n}+O\left(\mu_{k}^{1-2 / n}\right) .
$$

В соответствии с выдвинутой ранее гипотезой можно предположить, что в этом случае достаточное условие возникновения стационарных пространственно неоднородных решений будет иметь вид

$$
d_{1}<c \frac{\operatorname{Re}\left( \pm b_{n 1}\right)^{1 / n}}{\mu_{1}^{1-1 / n}}, \quad c=\text { const }>0 .
$$

\section{СПИСОК ЦИТИРОВАННОЙ ЛИТЕРАТУРЫ}

[1] Д. Хенри, Геометрическая теория полулинейных параболических уравнений, Мир, M., 1985.

[2] J. D. Murray, Mathematical Biology, Biomathematics, 19, Springer, Berlin, 1993.

[3] Дж. Марсден, М. Мак-Кракен, Бифуркация рождения изикла и ее приложения, Мир, М., 1980.

[4] С. Г. Михлин, Вариационные методы в математической физике, Наука, М., 1970.

[5] Т. Като, Теория возмущений линейных операторов, Мир, М., 1972. 
[6] М.И. Вишик, Л. А. Люстерник, "Решение некоторых задач о возмущении в случае матриц и самосопряженных и несамосопряженных дифференциальных уравнений. I", УМH, 15:3 (1960), 3-80.

[7] А. Ю. Колесов, Н. Х. Розов, "О существовании асимптотически большого числа устойчивых диссипативных структур в параболических системах с малой диффузией”, Тр. сем. им. И. Г. Петровского, 20, Изд-во Моск. ун-та, М., 1997, 3-26.

[8] А. С. Братусь, В.П. Посвянский, "Стационарные решения в замкнутой распределенной системе эволюции Эйгена-Шустера”, Дифферени. уравнения, 42:12 (2006), $1686-1698$.

\section{А. С. Братусь}

Московский государственный университет путей сообщения (МИИТ)

E-mail: applmath1miit@yandex.ru

\section{B. Caфpo}

Московский государственный университет путей сообщения (МИИТ)

E-mail: safromisha@rambler.ru
Поступило

05.06.2009

Исправленный вариант

25.03.2010 